\title{
Finer regularity of an entropy solution for 1-d scalar conservation laws with non uniform convex flux
}

\author{
Adimurthi(*) - Shyam Sundar Ghoshal (**) - G.D. Veerappa GoWda(***)
}

ABSTRACT - Consider a scalar conservation law in one space dimension with initial data in $L^{\infty}$. If the flux $f$ is in $C^{2}$ and locally uniformly convex, then for all $t>0$, the entropy solution is locally in BV (functions of bounded variation) in space variable. In this case it was shown in [5], that for all most every $t>0$, locally, the solution is in SBV (Special functions of bounded variations). Furthermore it was shown with an example that for almost everywhere in $t>0$ cannot be removed. This paper deals with the regularity of the entropy solutions of the strict convex $C^{1}$ flux $f$ which need not be in $C^{2}$ and locally uniformly convex. In this case, the entropy solution need not be locally in BV in space variable, but the composition with the derivative of the flux function is locally in BV. Here we prove that, this composition is locally is in SBV on all most every $t>0$. Furthermore we show that this is optimal.

Mathematics Subject Classification (2010). 35B65, 35L65, 35L67.

KEYwORDs. Hamilton-Jacobi equation, scalar conservation laws, characteristic lines, sbv functions, uniformly convex.

\section{Introduction}

Let $f: \mathbb{R} \rightarrow \mathbb{R}$ be a locally Lipschitz continuous function and $u_{0} \in$ $L^{\infty}(\mathbb{R})$. Consider the following scalar conservation law in one space di-

(*) Indirizzo dell'A.: Center for Applicable Mathematics, Tata Institute of Fundamental Research, PO Bag No. 6503, Bangalore-560065, India.

E-mail: aditi@math.tifrbng.res.in

(**) Indirizzo dell'A.: Laboratoire de Mathématiques, Université de FrancheComté, 25030 Besançon, France.

E-mail: ssghosha@univ-fcomte.fr

The second author would like to thank French ANR project "CoToCoLa" for the support.

(***) Indirizzo dell'A.: Center for Applicable Mathematics, Tata Institute of Fundamental Research, PO Bag No. 6503, Bangalore-560065, India.

E-mail: gowda@math.tifrbng.res.in 
mension

$$
\begin{array}{rlrl}
\frac{\partial u}{\partial t}+\frac{\partial}{\partial x} f(u) & =0 & \text { if } \quad & x \in \mathbb{R}, t>0 . \\
u(x, 0) & =u_{0}(x) \quad \text { if } \quad x \in \mathbb{R} .
\end{array}
$$

A function $u \in L^{\infty}\left(\mathbb{R} \times \mathbb{R}_{+}\right)$is said to be a solution of (1.1) if $u$ is a weak solution of (1.1) satisfying Kruzkov [14] entropy condition. By using vanishing viscosity, Kruzkov [14] proved that there exists a unique entropy solution to (1.1).

Under the convexity assumption on the flux, Lax and Oleinik [12] have obtained an explicit formula for the solution. In order to state their result, let us recall some definitions and their properties of convex functions [4].

Let $f: \mathbb{R} \rightarrow \mathbb{R}$ be a convex function. Then

(i) $f$ is said to be a strictly convex function if for all $x \neq y \alpha \in(0,1)$,

$$
f(\alpha x+(1-\alpha) y)<\alpha f(x)+(1-\alpha) f(y) .
$$

(ii) $f \in C^{2}(\mathbb{R})$ is said to be locally uniformly convex if for all compact set $K \subset \mathbb{R}$, there exist a constant $C(K)>0$ such that $f^{\prime \prime}(x) \geq C(K)$ for all $x \in K$. It is said to be uniformly convex in $\mathbb{R}$ if there exist $C>0$ such that $f^{\prime \prime}(x) \geq C$ for all $x \in \mathbb{R}$.

(iii) $f$ is said to be of super linear growth if

$$
\lim _{|u| \rightarrow \infty} \frac{f(u)}{|u|}=\infty .
$$

(iv)Associate to a convex function $f$, let $f^{*}$ be its convex dual defined by

$$
f^{*}(p)=\sup _{q}\{p q-f(q)\} .
$$

Then for any $q \in \mathbb{R}$,

$$
\lim _{|p| \rightarrow \infty} \frac{f^{*}(p)}{|p|} \geq \frac{\lim _{|p| \rightarrow \infty}}{|q|}\left(q \frac{p}{|p|}-\frac{f(q)}{|p|}\right)=\lim _{|p| \rightarrow \infty} q \frac{p}{|p|}
$$

since $q$ is arbitrary and letting $|q| \rightarrow \infty$ to obtain

$$
\lim _{|p| \rightarrow \infty} \frac{f^{*}(p)}{|p|}=\infty
$$

(v) Let $f$ be in $C^{1}(\mathbb{R})$ and strictly convex. Then $f^{\prime}$ is a strictly increasing function. Furthermore, it can be shown that if $f$ is of super linear growth, then $f=f^{* *},\left(f^{*}\right)^{\prime}=\left(f^{\prime}\right)^{-1}$. Therefore $f^{*}$ is in $C^{1}(\mathbb{R})$ with super linear 
growth and is strictly convex. With these preliminaries, we recall the following (see [12]):

THEOREM 1.1. (Lax and Olenik) Assume that $f$ is in $C^{1}(\mathbb{R})$, strictly convex and of super linear growth. Then there exist a function $y(x, t)$ such that $x \mapsto y(x, t)$ is a non decreasing function and for all $t>0$, a.e. $x \in \mathbb{R}$, the solution $u$ of (1.1) is explicitly given by

$$
f^{\prime}(u(x, t))=\frac{x-y(x, t)}{t} .
$$

As an immediate consequence of this theorem, we have

(1) For each $t>0, x \mapsto f^{\prime}(u(x, t)) \in B V_{\text {loc }}(\mathbb{R})$.

(2) If $f \in C^{2}$ and locally uniformly convex, then for all $t>0, x \mapsto u(x, t)$ is in $B V_{l o c}(\mathbb{R})$.

In this context, Alberto Bressan raised the following question.

Problem 1. Assume that for all $t>0, x \mapsto u(x, t) \in B V_{l o c}(\mathbb{R})$, then is it in $S B V_{\text {loc }}(\mathbb{R})$ ?

Under the suitable condition on $f$, Ambrosio-De Lellis [5] proved the following

THEOREM 1.2. (Ambrosio-De Lellis) Assume further that $f \in C^{2}(\mathbb{R})$ and locally uniformly convex. Then there exist a countable set $S \subset(0, \infty)$ such that for all $t \notin S, x \mapsto u(x, t) \in S B V_{l o c}(\mathbb{R})$. Furthermore, they gave an example for which $S \neq \phi$.

Observe that from the explicit formula, if $f^{\prime-1}$ is a locally Lipschitz continuous, then the solution is in $B V_{l o c}(\mathbb{R})$ in space variable which is guaranteed if $f$ is $C^{2}$ and locally uniformly convex. This is what assumed in [5]. In [7], the condition of locally uniformly convexity has been relaxed to prove the same result as in [5] provided the zero set of $f^{\prime \prime}$ is countable and $u$ is in $B V_{l o c}$. In [15] even the convexity condition is relaxed under the hypothesis that $f$ is $C^{2}$ and the zero set of $f^{\prime \prime}$ is countable. For further generalization see [6], [8], [9], [10], [16].

Now the natural question is "What happens if $f \in C^{1}$ and satisfying the hypothesis of Lax-Olenik Theorem?" In this case, the problem of Bressan can be reformulated as follows. 


\section{Problem 2.}

(i) Does $x \mapsto u(x, t) \in B V_{l o c}(\mathbb{R})$ for all $t>0$.

(ii) Does $x \mapsto f^{\prime}(u(x, t)) \in S B V_{l o c}(\mathbb{R})$ for a.e. $t>0$.

(iii) If (ii) is true, does there exist a $u_{0} \in L^{\infty}(\mathbb{R})$ and a countable set $S=\left\{t_{n}\right\}$ such that $f^{\prime}\left(u\left(\cdot, t_{n}\right)\right) \notin S B V_{l o c}(\mathbb{R})$ for all $n$.

In view of (1.2), the main result of this paper is to show that the Problem 2 has an affirmative answer and we have the following

THEOREM 1.3. Let $f$ satisfies the hypothesis of Theorem 1.1 and $u$ be the solution of (1.1). Then

(1) There exist a countable set $S \subset(0, \infty)$ such that for all $t \notin S, x \mapsto f^{\prime}(u(x, t)) \in S B V_{l o c}(\mathbb{R})$.

(2) Assume that $f(0)=f^{\prime}(0)=0$ and there exist $c>0$ and $0<\gamma<1$ such that for all $\alpha \leq 0 \leq \beta, f$ satisfies

$$
f^{\prime^{-1}}(\beta)-f^{\prime^{-1}}(\alpha) \geq c(\beta-\alpha)^{\gamma}
$$

Then there exist a $u_{0} \in L^{\infty}(\mathbb{R})$ such that for all $t>0, x \mapsto u(x, t) \notin$ $B V_{\text {loc }}(\mathbb{R})$.

(3) There exist a $u_{0} \in L^{\infty}(\mathbb{R})$ and a decreasing sequence $\left\{t_{n}\right\}$ such that $x \mapsto f^{\prime}\left(u\left(x, t_{n}\right)\right) \notin S B V_{l o c}(\mathbb{R})$.

For (1), we follow the similar path as in [5]. The idea in the proof of (2) lies in the construction of asymptotically single shock packets as in [1]. The proof of (3) lies in the backward construction (see Appendix) as in [2], [3].

REMARK 1.4. There are many fluxes satisfying the hypothesis of (2). For example, let $p>2$, then $f(u)=\frac{|u|^{p}}{p}$ is one of such function.

\section{Preliminaries}

Let $f$ be as in Theorem 1.1. Let $u_{0} \in L^{\infty}(\mathbb{R})$. Let $A \in \mathbb{R}$ and define

$$
\begin{gathered}
v_{0}(x)=\int_{A}^{x} u_{0}(\theta) d \theta \\
f^{*}(p)=\max _{q}\{p q-f(q)\}
\end{gathered}
$$


the convex dual of $f$. Then from the hypothesis of $f$, it follows that $f^{*} \in C^{1}(\mathbb{R})$, strictly convex and having super linear growth.

Let $t>0, x \in \mathbb{R}$, define

$$
v(x, t)=\min _{y}\left\{v_{0}(y)+t f^{*}\left(\frac{x-y}{t}\right)\right\} .
$$

Then we have the following (see [12])

TheOREM 2.1. (Hopf): $v \in \operatorname{Lip}\left(\overline{\mathbb{R} \times \mathbb{R}_{+}}\right)$and is the unique viscosity solution of the following Hamilton Jacobi equation.

$$
\begin{aligned}
\frac{\partial v}{\partial t}+f\left(\frac{\partial v}{\partial x}\right) & =0 & & \text { if } x \in \mathbb{R}, t>0 \\
v(x, 0) & =v_{0}(x) & & \text { if } x \in \mathbb{R}
\end{aligned}
$$

furthermore it satisfies

(i) Dynamic programming principle: Let $0 \leq s<t$ : then

$$
v(x, t)=\min _{y \in \mathbb{R}}\left\{v(y, s)+(t-s) f^{*}\left(\frac{x-y}{t-s}\right)\right\}
$$

(ii) There exist a $M>0$ depending on $u_{0}$ and $f^{*}$ such that for all minimizers of $y$ of (2.5)

$$
\left|\frac{x-y}{t-s}\right| \leq M
$$

Next we define the different notions of characteristics and their properties without proof.

Definition 2.2. Let $0 \leq s<t ; x \in \mathbb{R}$. Then

(i) Characteristic sets $\operatorname{ch}(x, s, t), \operatorname{ch}(x, t)$ are defined by

$$
\begin{aligned}
\operatorname{ch}(x, s, t) & =\{y \in \mathbb{R} ; \quad y \text { is a minimizer in 2.5) }\} \\
\operatorname{ch}(x, t) & =\operatorname{ch}(x, 0, t) .
\end{aligned}
$$

(ii) $y \in \operatorname{ch}(x, s, t)$ is called a characteristic point and the line joining $(x, t)$ and $(y, s)$ is called a characteristic line segment.

(iii) Extreme characteristic points

$$
\begin{aligned}
& y_{+}(x, s, t)=\max \{y \in \operatorname{ch}(x, s, t)\} \\
& y_{-}(x, s, t)=\min \{y \in \operatorname{ch}(x, s, t)\} \\
& y_{ \pm}(x, t)=y_{ \pm}(x, 0, t)
\end{aligned}
$$


(iv) The characteristic line segment associated to $y_{ \pm}(x, s, t)$ are called extreme characteristic line segments.

Definition 2.3. (proper intersection) Let $0 \leq s_{i}<t_{i}$ and for $i=1,2, r_{i}=\left[s_{i}, t_{i}\right] \rightarrow \mathbb{R}$ be affine maps. Then $r_{1}$ and $r_{2}$ are said to intersect properly if there exist a $\theta_{0} \in\left(\max \left(s_{1}, s_{2}\right), \min \left(t_{1}, t_{2}\right)\right)$ such that $r_{1}\left(\theta_{0}\right)=r_{2}\left(\theta_{0}\right)$.

\section{Properties of Characteristics (see [12])}

(1) No two distinct characteristic line segments intersect properly.

(2) Let $y_{1} \in \operatorname{ch}(x, s, t)$ and $y_{2} \in \operatorname{ch}\left(y_{1}, s\right)$. Then $y_{2} \in \operatorname{ch}(x, t)$ and $(x, t)$, $\left(y_{1}, s\right),\left(y_{2}, 0\right)$ are collinear. That is

$$
\frac{x-y_{2}}{t}=\frac{x-y_{1}}{t-s}=\frac{y_{1}-y_{2}}{s} .
$$

(3) $x \mapsto y_{ \pm}(x, s, t)$ are non decreasing functions and at points of continuity $x$ of $y_{+}(\cdot, s, t)$

$$
y_{+}(x, s, t)=y_{-}(x, s, t) .
$$

(4) Lax-Oleinik explicit formula: Let $u(x, t)=\frac{\partial v}{\partial x}(x, t)$, then $u$ is the unique solution of (1.1) satisfying

$$
f^{\prime}(u(x, t))=\frac{x-y_{+}(x, s, t)}{t-s} \quad \text { for } \text { a.e. } x \in \mathbb{R} .
$$

Definition 2.4 (characteristic curve). Let $0 \leq s<t$ and $\alpha \in \mathbb{R}$. Define the characteristic curve $\xi(t, s, \alpha)$ passing through $\alpha$ by

$$
\xi(t, s, \alpha)=\inf \left\{\xi \in \mathbb{R} ; y_{-}(\xi, s, t) \geq \alpha\right\} \text {. }
$$

Then we have the following

LEMMA 2.5. Let $0 \leq s<t$, then

(1) Let $x_{n} \rightarrow x_{0}, y_{n} \in \operatorname{ch}\left(x_{n}, s, t\right)$ such that $y_{n} \rightarrow y_{0}$. Then $y_{0} \in \operatorname{ch}\left(x_{0}, s, t\right)$.

(2) $\lim _{x \uparrow x_{0}} y_{+}(x, s, t)=y_{-}\left(x_{0}, s, t\right)$ and $\lim _{x \downarrow x_{0}} y_{-}(x, s, t)=y_{+}\left(x_{0}, s, t\right)$.

$$
\begin{aligned}
& y_{+}(x, s, t)<y_{-}(\xi(t, s, \alpha), s, t) \text { if } \quad x<\xi(t, s, \alpha) \\
& \text { (3) (2.12) } \quad y_{-}(x, s, t) \geq y_{+}(\xi(t, s, \alpha), s, t) \text { if } \quad x>\xi(t, s, \alpha) \\
& \text { (2.13) } y_{-}(\xi(t, s, \alpha), s, t) \leq \alpha \leq y_{+}(\xi(t, s, \alpha), s, t) .
\end{aligned}
$$


(4) $t \mapsto \xi(t, s, \alpha)$ is a Lipschitz continuous function with

$$
\lim _{t \rightarrow s} \xi(t, s, \alpha)=\alpha .
$$

Proof. From (2.5), we have for all $y$,

$$
\begin{aligned}
v\left(x_{n}, t\right) & =v\left(y_{n}, s\right)+(t-s) f^{*}\left(\frac{x_{n}-y_{n}}{t-s}\right) \\
& \leq v(y, s)+(t-s) f^{*}\left(\frac{x_{n}-y}{t-s}\right) .
\end{aligned}
$$

Since $v$ is continuous, hence letting $n \rightarrow \infty$ to obtain

$$
\begin{aligned}
v\left(x_{0}, t\right) & =v\left(y_{0}, s\right)+(t-s) f^{*}\left(\frac{x_{0}-y_{0}}{t-s}\right) \\
& \leq v(y, s)+(t-s) f^{*}\left(\frac{x_{0}-y}{t-s}\right) .
\end{aligned}
$$

Hence $y_{0} \in C h\left(x_{0}, x, t\right)$. This proves (1).

From the non proper intersection of characteristic line segments it follows that if $x<x_{0}$, then $y_{+}(x, s, t) \leq y_{-}\left(x_{0}, s, t\right)$. Let $x_{n} \uparrow x_{0}$ and $y_{+}\left(x_{n}, s, t\right) \rightarrow y_{0}$. Then from (1), $y_{0} \in \operatorname{ch}\left(x_{0}, s, t\right)$ and hence

$$
y_{-}\left(x_{0}, s, t\right) \leq y_{0}=\lim y_{+}\left(x_{n}, s, t\right) \leq y_{-}\left(x_{0}, s, t\right) .
$$

This proves (2).

From (2) and definition of $\xi(\cdot, s, \alpha)$ gives

$$
\begin{array}{lll}
y_{+}(x, s, t)<y_{-}(\xi(t, s, \alpha), s, t) & \text { if } \quad x<\xi(t, s, \alpha) \\
y_{-}(x, s, t) \geq y_{+}(\xi(t, s, \alpha), s, t) & \text { if } \quad x>\xi(t, s, \alpha) .
\end{array}
$$

Choose a sequence $x_{n}<\xi(t, s, \alpha)$ such that $x_{n} \uparrow \xi(t, s, \alpha)$ and choose a $n_{0}>0$ such that for all $n>n_{0}$,

$$
y_{-}\left(x_{n}, s, t\right)=y_{+}\left(x_{n}, s, t\right) .
$$

Since $y_{-}\left(x_{n}, s, t\right)<\alpha$ and hence from (2) we have

$$
\begin{aligned}
y_{-}(\xi(t, s, \alpha), s, t) & =\lim _{x_{n} \uparrow \xi(t, s, \alpha)} y_{+}\left(x_{n}, s, t\right) \\
& =\lim _{x_{n} \uparrow \xi(t, s, \alpha)} y_{-}\left(x_{n}, s, t\right) \\
& \leq \alpha .
\end{aligned}
$$

Similarly other inequality holds and this proves (3).

Let $s \leq t_{1}<t_{2}$ and let $y_{ \pm}$be the extreme characteristic points and $r_{ \pm}(\theta)$ be the characteristic line segments at $\left(\xi\left(t_{2}, s, \alpha\right), t_{2}\right)$. Then $\gamma_{ \pm}$are given by

$$
\gamma_{ \pm}(\theta)=\xi\left(t_{2}, s, \alpha\right)+\left(\theta-t_{2}\right) \frac{\xi\left(t_{2}, s, \alpha\right)-y_{ \pm}}{t_{2}-s .}
$$


From (2.13) and non proper intersection of characteristic line segments, it follows that for all $s<t_{1}<t_{2}$,

$$
\gamma_{-}\left(t_{1}\right) \leq \xi\left(t_{1}, s, \alpha\right) \leq \gamma_{+}\left(t_{1}\right) .
$$

Hence from (2.6), (2.15) and (2.16) we have

$$
\begin{aligned}
& \left|\xi\left(t_{1}, s, \alpha\right)-\xi\left(t_{2}, s, \alpha\right)\right| \leq 2 M\left|t_{2}-t_{1}\right| \\
& \left|\xi(t, s, \alpha)-y_{ \pm}\right| \leq M|t-s|
\end{aligned}
$$

which proves (2.15) and hence (4). This proves the Lemma.

We follow the same notations as in [5]. For $t>0, x \in \mathbb{R}, \theta \in[0, t]$ define

$$
\begin{array}{ll}
m_{ \pm}(x, t) & =\frac{x_{-} y_{ \pm}(x, t)}{t} \\
\gamma_{ \pm}(\theta) & =x+m_{ \pm}(x, t)(\theta-t) \\
I(x, t) & =\left(y_{-}(x, t), y_{+}(x, t)\right) \\
C(x, t) & =\left\{(\xi, \theta) ; 0<\theta<t, \gamma_{-}(\theta)<\xi<\gamma_{+}(\theta)\right\} \\
D(t) & =\left\{x \in \mathbb{R} ; y_{+}(x, t) \text { has a jump at } x\right\} \\
I(t) & =\bigcup_{x \in D(t)} I(x, t) \\
c(t) & =\bigcup_{x \in D(t)} C(x, t) \\
F(t) & =|I(t)| .
\end{array}
$$

Where $|I(t)|$ denotes the Lebesgue measure of $I(t)$. Then we have the following

Lemma 2.6. Let $0<s<t, \xi, x_{1}, x_{2} \in \mathbb{R} ; x_{1} \neq x_{2}$ and $(\xi, s) \in C(x, t)$. Then

$$
\begin{gathered}
I\left(x_{1}, t\right) \cap I\left(x_{2}, t\right)=\phi, C\left(x_{1}, t\right) \cap C\left(x_{2}, t\right)=\phi \\
I(\xi, s) \subset I(x, t), C(\xi, s) \subset C(x, t) \\
I(s) \subset I(t)
\end{gathered}
$$

Proof. (2.17) and (2.18) follows from the non proper intersection of characteristic line segments. Let $x \in D(s), \xi_{0}=\xi(t, s, x)$ and $y_{ \pm}=$ $y_{ \pm}\left(\xi_{0}, s, t\right)$. Then from (2.13), $y_{-} \leq x \leq y_{+}$. Suppose $y_{-}=x$, then from (2.7), the points $\left(\xi_{0}, t\right),(x, s),\left(y_{+}(x, s), 0\right)$ lie on a straight line and also the points $\left(\xi_{0}, t\right),(x, s),\left(y_{-}(x, s), 0\right)$ lie on a straight line. Hence $y_{+}(x, s)=y_{-}(x, s)$ 
which contradicts the fact that $x \in D(s)$. Hence $y_{-}<x<y_{+}$and this implies $y_{-}\left(\xi_{0}, t\right) \leq y_{-}(x, s)<y_{+}(x, s) \leq y_{+}\left(\xi_{0}, t\right)$. This proves (2.19) and hence the Lemma.

Lemma 2.7. Let $t>0, x \in \mathbb{R}$. Assume that at $(x, t), y_{ \pm}(x, t)$ satisfies the following hypothesis.

(i) $y_{+}(x, t)=y_{-}(x, t)$.

(ii) Let $\eta>0, x-\eta \notin D(t)$. Assume one of the following holds

(2.20) $\lim _{\substack{\eta \rightarrow 0 \\ x-\eta \notin D(t)}} \frac{y_{+}(x, t)-y_{+}(x-\eta, t)}{\eta}=\infty, \lim _{\substack{\eta \rightarrow 0 \\ x+\eta \notin D(t)}} \frac{y_{+}(x+\eta, t)-y_{+}(x, t)}{\eta}=\infty$.

Then for all $t<\tau$ and $z \in \mathbb{R}$,

$$
x \notin \operatorname{ch}(z, t, \tau) .
$$

Proof. Suppose not, then there exist a $z_{0} \in \mathbb{R}, \tau_{0}>t$ such that $x \in \operatorname{ch}\left(z_{0}, t, \tau_{0}\right)$. Since $y_{+}(x, t) \in \operatorname{ch}(x, t)$ and hence from (2.7) we have

$$
\frac{x-y_{+}(x, t)}{t}=\frac{z_{0}-x}{\tau_{0}-t} .
$$

Let $\alpha=\frac{z_{0}-x}{\tau_{0}-t}$ be this common value. Let

$$
h(z)=\int_{A}^{z} u(\theta, t) d \theta+\left(\tau_{0}-t\right) f^{*}\left(\frac{z_{0}-z}{\tau_{0}-t}\right) .
$$

Then from (2.5), we have

$$
h(x)=\min _{z \in \mathbb{R}} h(z) .
$$

Without loss of generality we can assume that

$$
\lim _{\substack{n \rightarrow 0 \\ x \rightarrow n \notin D(t)}} \frac{y_{+}(x, t)-y_{+}(x-\eta, t)}{\eta}=\infty
$$

and similar argument hold if the other limit holds. Let $\wedge>1+\frac{t}{\tau_{0}-t}$ and $\eta_{0}>0$ such that for all $\theta \in\left(0, \eta_{0}\right) \backslash D(t)$

$$
-y_{+}(x-\theta, t)>\wedge \theta-y_{+}(x, t)
$$

Since $f^{*^{\prime}}=\left(f^{\prime}\right)^{-1}$, is a strictly increasing function, hence for $\eta \in\left(0, \eta_{0}\right)$, and from (2.9), (2.2) and (2.25) we have 


$$
\begin{aligned}
h(x)-h(x-\eta)= & \int_{A}^{x} u(\theta, t) d \theta-\int_{A}^{x-\eta} u(\theta, t) d \theta \\
& +\left(\tau_{0}-t\right) f^{*}\left(\frac{z_{0}-x}{\tau_{0}-t}\right)-\left(\tau_{0}-t\right) f^{*}\left(\frac{z_{0}-x+\eta}{\tau_{0}-t}\right) \\
= & \int_{0}^{\eta} u(x-\theta, t) d \theta+\left(\tau_{0}-t\right)\left\{f^{*}(\alpha)-f^{*}\left(\alpha+\frac{\eta}{\tau_{0}-t}\right)\right\} \\
= & \int_{0}^{\eta} f^{*^{\prime}}\left(\frac{x-\theta-y_{+}(x-\theta, t)}{t}\right) d \theta+\left(\tau_{0}-t\right)\left\{f^{*}(\alpha)-f^{*}\left(\alpha+\frac{\eta}{\tau_{0}-t}\right)\right\} \\
\geq & \int_{0}^{\eta} f^{*^{\prime}}\left(\frac{x-y_{+}(x, t)}{t}+\left(\frac{\wedge-1}{t}\right) \theta\right) d \theta \\
+ & \left.\left(\tau_{0}-t\right)\left\{f^{*}(\alpha)-f^{*} \alpha+\frac{\eta}{\tau_{0}-t}\right)\right\} \\
+ & \left(\frac{t}{\wedge-1}\right)\left\{f^{*}\left(\alpha+\left(\frac{\wedge-1}{t}\right) \eta\right)-f^{*}(\alpha)\right\} \\
& \\
& \left(\tau_{0}-t\right)\left\{f^{*}(\alpha)-f^{*}\left(\alpha+\frac{\eta}{\tau_{0}-t}\right)\right\} .
\end{aligned}
$$

Let $g(s)=f^{*}(\alpha+s)-f^{*}(\alpha)$, then $g(0)=0$ and $g$ is a strictly convex function. Let $a>b>0$, then we have $g(b)=g\left(\frac{b}{a} a\right)<\left(1-\frac{b}{a}\right) g(0)+$ $\frac{b}{a} g(a)=\frac{b}{a} g(a)$. Hence $\frac{g(b)}{b}<\frac{g(a)}{a}$. Let $\eta>0, a=\left(\frac{\wedge-1}{t}\right) \eta>\frac{n}{\tau_{0}-t}=b$. Therefore from $\frac{g(b)}{b}<\frac{g(a)}{a}$ implies that

$$
\frac{t}{\eta(\wedge-1)}\left[f^{*}\left(\alpha+\left(\frac{\wedge-1}{t}\right) \eta\right)-f^{*}(\alpha)\right]>\frac{\tau_{0}-t}{\eta}\left[f^{*}\left(\alpha+\frac{\eta}{\tau_{0}-t}\right)-f^{*}(\alpha)\right]
$$

This implies that $h(x)>h(x-\eta)$ contradicting (2.24). This proves the Lemma.

Corollary 2.8. Let $(x, t)$ satisfies the hypothesis of lemma (2.3) and $\tau>t$. Let $\xi_{0}=\xi(\tau, t, x)$, then $(x, t) \in C\left(\xi_{0}, \tau\right)$. 
Proof. From (2.13) and (2.21) it follows that

$$
y_{-}\left(\xi_{0}, t, \tau\right)<x<y_{+}\left(\xi_{0}, t, \tau\right)
$$

From (2.7) it follows that $\left(y_{-}\left(\xi_{0}, t, \tau\right), y_{+}\left(\xi_{0}, t, \tau\right)\right) \times\{t\} \subset C\left(\xi_{0}, \tau\right)$ and hence $(x, t) \in C\left(\xi_{0}, \tau\right)$. This proves the corollary.

Proof of Theorem 1.3. In view of the finite speed propagation, without loss of generality, assume that $u_{0}$ is as follows:

$$
u_{0}(x)=\left\{\begin{array}{lll}
u_{-} & \text {if } & x<A \\
\bar{u}_{0}(x) & \text { if } & x \in(A, B) \\
u_{+} & \text {if } & x>B
\end{array}\right.
$$

where $u_{ \pm}$are constants and $\bar{u}_{0} \in L^{\infty}(A, B)$. Let

$$
\begin{gathered}
m=\inf u_{0}, \quad M=\sup u_{0} \\
\alpha(t)=A+t f^{\prime}(m), \beta(t)=B+t f^{\prime}(M)
\end{gathered}
$$

then it follows that

$$
u(x, t)=\left\{\begin{array}{lll}
u_{-} & \text {if } & x<\alpha(t) \\
u_{+} & \text {if } & x>\beta(t)
\end{array}\right.
$$

Hence from (2.9)

$$
y_{+}(x, t)=y_{-}(x, t)=\left\{\begin{array}{lll}
x-t f^{\prime}\left(u_{-}\right) & \text {if } & x<\alpha(t) \\
x-t f^{\prime}\left(u_{+}\right) & \text {if } & x>\beta(t) .
\end{array}\right.
$$

As a consequence of this we have

LEMma 3.9. For $t>0, F(t)<\infty$ and is a nondecreasing function.

Proof. From (3.4), $D(t) \subset[\alpha(t), \beta(t)]$ and hence

$$
F(t)=|I(t)|=\sum_{x \in D(t)}\left(y_{+}(x, t)-y_{-}(x, t)\right) \leq \beta(t)-\alpha(t)<\infty .
$$

From (2.19), $F$ is a non decreasing function and this proves the Lemma.

Let $d y(\cdot, t)$ be the Distributional derivatives of $x \mapsto y(x, t)$. Then $d y(\cdot, t)$ is a non negative measure. Decompose $d y(\cdot, t)$ as follows:

$$
d y(\cdot, t)=f_{t} d x+d v_{t}+d \mu_{t}
$$

where the measures are mutually singular, with $f_{t} \in L_{l o c}^{1}(\mathbb{R}), d v_{t}$ is the jump 
part and $\mu_{t}$ is the Cantor part (see [5]). From (3.4) it follows that the support of $d \mu_{t}, d v_{t}$ are in $[\alpha(t), \beta(t)]$ and $d y(., t)=d x$ for $x \notin[\alpha(t), \beta(t)]$.

For a measurable set $A \subset \mathbb{R}$, denote $|A|$ to be its Lebesgue measure. Then from the Besicovitch differentiation Theorem (see [13]) it follows that there exists a set $E_{t} \subset[\alpha(t), \beta(t)]$ with the property:

$$
\left|E_{t}\right|=0, E_{t} \cap D_{t}=\phi, \mu_{t}(\mathbb{R})=\mu_{t}\left(E_{t}\right)
$$

and for $\mu_{t}$ a.e. $x \in E_{t}$

$$
\lim _{\eta \rightarrow 0, x \pm \eta \notin D(t)} \frac{2 \eta+v_{t}[x-\eta, x+\eta]}{\mu_{t}([x-\eta, x+\eta])}=0 .
$$

To prove theorem (1.3), we need to replace the symmetric limit by one sided limit in (3.6) and is given by the following

LEMMA 3.10. For $\mu_{t}$ a.e. $x \in E_{t}$, we have

$$
\infty=\lim _{\eta \rightarrow 0, x-\eta \notin D(t)} \frac{y_{+}(x, t)-y_{+}(x-\eta, t)}{\eta}=\lim _{\eta \rightarrow 0, x+\eta \notin D(t)} \frac{y_{+}(x+\eta, t)-y_{+}(x, t)}{\eta}
$$

To prove this lemma, one uses a covering lemma different from that of Besicovitch covering lemma. For the sake of completeness, proof of this has been given in the appendix.

LEMMA 3.11. Let $t>0$, then there exist a constant $C>0$ such that for all $\tau>t$,

$$
F(\tau)-F(t) \geq C \mu_{t}\left(E_{t}\right)
$$

Proof. For $x \in \mathbb{R}$, denote $\xi_{0}(x)=\xi(\tau, t, x)$. Let $x \in E_{t}$ satisfying (3.5) and (3.6). Then from (3.6), (3.7), corollary (2.1), choose $\eta(x)$ small such that $x \pm \eta(x) \notin D(t)$ and

$$
\begin{gathered}
{[x-\eta(x), x+\eta(x)] \times\{t\} \subset C\left(\xi_{0}(x), \tau\right)} \\
v_{t}([x-\eta(x), x+\eta(x)]) \leq \frac{1}{2} \mu_{t}([x-\eta(x), x+\eta(x)])
\end{gathered}
$$

Since

$$
v_{t}([x-\eta(x), x+\eta(x)])=\sum_{z \in[x-\eta(x), x+n(x)] \cap D(t)}|I(z, t)|
$$


therefore from (2.17), (2.18), (3.9) and (3.10), we have

$$
\begin{aligned}
& \left|I\left(\xi_{0}(x), \tau\right)\right|-\sum_{(\xi, t) \in C\left(\xi_{0}(x), \tau\right) \cap(D(t) \times\{t\})}|I(\xi, t)| \\
& \geq\left(y_{+}(x+\eta(x), t)-y_{-}(x-\eta(x), t)\right)-\sum_{z \in[x-\eta(x), x+\eta(x)] \cap D(t) \times\{t\})} I(z, t) \\
& \geq \mu_{t}\left([x+\eta(x), x-\eta(x)]-v_{t}([x-\eta(x), x+\eta(x)])\right. \\
& \geq \frac{1}{2} \mu_{t}([x-\eta(x), x+\eta(x)]) .
\end{aligned}
$$

Now $\{[x-\eta(x), x+\eta(x)]\}_{x \in E_{t}}$ covers $E_{t}$ and hence by Besicovitch covering Lemma, there exists a countable disjoint set of intervals $\left\{\left[x_{j}-\eta_{j}, x_{j}+\eta_{j}\right]\right\}$ which satisfies (3.11) and

$$
\sum_{j} \mu_{j}\left[x_{j}-\eta_{j}, x_{j}+\eta_{j}\right] \geq C \mu_{t}\left(E_{t}\right)
$$

for some $C>0$. Therefore

$$
\begin{aligned}
F(\tau)-F(t) & \left.\geq \sum_{j} \mid I\left(\xi_{0}\left(x_{j}\right)\right), \tau\right)\left|-\sum_{j} \sum_{(\xi, t) \in C\left(\left(\xi_{0}\left(x_{j}\right), \tau\right) \cap(D(t) \times\{t\})\right.}\right| I(\xi, t) \mid \\
& \geq \frac{1}{2} \sum_{j} \mu_{t}\left[\left(x_{j}-\eta_{j}, x_{j}+\eta_{j}\right]\right) \\
& \geq \frac{C}{2} \mu_{t}\left(E_{t}\right) .
\end{aligned}
$$

This proves (3.8) and hence the Lemma.

Before going to the next Lemma we need the following:

Let $f$ be as in Theorem (1.3) with $f(0)=f^{\prime}(0)=0$. Since $f(\theta) / \theta$ is a strictly increasing function we therefore have $\theta f^{\prime}(\theta)-f(\theta)>0$ for $\theta \neq 0$. Since $f(0)=f^{\prime}(0)=0$, hence choose $\alpha<0$ and $\beta_{0}>0$ such that

$$
\beta_{0} f^{\prime}\left(\beta_{0}\right)-f\left(\beta_{0}\right)>\alpha f^{\prime}(\alpha)-f(\alpha)
$$

and let $g(\theta)=\theta f^{\prime}(\theta)-f(\theta)-\alpha f^{\prime}(\alpha)+f(\alpha)$. Then $g(0)<0$ and $g\left(\beta_{0}\right)>0$. Hence there exist a $\beta \in\left[0, \beta_{0}\right)$ such that $g(\beta)=0$. That is there exist $\alpha<0<\beta$ such that

$$
\beta f^{\prime}(\beta)-\alpha f^{\prime}(\alpha)=f(\beta)-f(\alpha)>0
$$

Let $\alpha<0<\beta$ be chosen as above and $m=\frac{f(\beta)-f(\alpha)}{\beta-\alpha}$. Let $A<B$, $\gamma_{1}(t)=A+t f^{\prime}(\beta), \gamma_{2}(t)=B+t f^{\prime}(\alpha)$ and $\gamma_{1}$, and $\gamma_{2}$ intersect at 
$T=\frac{B-A}{f^{\prime}(\beta)-f^{\prime}(\alpha)}$. Clearly if $X=\gamma_{1}(T)=\gamma_{2}(T)$, then $A<X<B$. Let $C=A+T\left(f^{\prime}(\beta)-m\right)=B+T\left(f^{\prime}(\alpha)-m\right)$ and $\gamma_{3}(t)=C+t m$ then by convexity, $A<C<B$ and $\gamma_{3}(T)=X$. Furthermore we have the following identity

$$
\begin{aligned}
\beta(C-A)+\alpha(B-C) & =T\left[\beta\left(f^{\prime}(\beta)-m\right)-\alpha\left(f^{\prime}(\alpha)-m\right)\right] \\
& =T\left[\beta f^{\prime}(\beta)-\alpha f^{\prime}(\alpha)+f(\alpha)-f(\beta)\right] \\
& =0
\end{aligned}
$$

Now define $u_{0, A, B}$ by

$$
u_{0, A, B}(x)=\left\{\begin{array}{lll}
0 & \text { if } & x \notin[A, B] \\
\beta & \text { if } & A<x<C \\
\alpha & \text { if } & C<x<B
\end{array}\right.
$$

Then from (3.13), we have

$$
\int_{A}^{B} u_{0, A, B}(x) d x=\beta(C-A)+\alpha(B-C)=0
$$

Let $u_{A, B}$ be the solution of (1.1) with initial data $u_{0, A, B}$. Then from the choice of $\alpha, \beta$ and $C$ for $0<t<T, \quad u_{A, B}$ is given by

$$
u_{A, B}(x, t)=\left\{\begin{array}{lll}
0 & \text { if } & x \notin[A, B] \\
f^{\prime-1}\left(\frac{x-A}{t}\right) & \text { if } & A<x<\gamma_{1}(t) \\
\beta & \text { if } & \gamma_{1}(t)<x<\gamma_{3}(t) \\
\alpha & \text { if } & \gamma_{3}(t)<x<\gamma_{2}(t) \\
f^{\prime-1}\left(\frac{x-B}{t}\right) & \text { if } & \gamma_{3}(t)<x<B
\end{array}\right.
$$

Then we have the following

LEMma 3.12. Under the notations as above, let $\xi(t)=\xi(t, 0, C)$ be the characteristic curve passing through $C$ and defined as in (2.10) for the solution $u_{A, B}$. Then for all $t>0$.

$$
\begin{gathered}
\xi(t)=\gamma_{3}(t) \quad \text { if } t<T \\
A<\xi(t)<B \quad \text { if } t>T .
\end{gathered}
$$


For $t>T, u_{A, B}$ is given by

$$
u_{A, B}(x, t)= \begin{cases}0 & \text { if } \quad x \notin(A, B) \\ f^{\prime^{-1}}\left(\frac{x-A}{t}\right) & \text { if } \quad A<x<\xi(t) \\ f^{\prime^{-1}}\left(\frac{x-B}{t}\right) & \text { if } \quad \xi(t)<x<B .\end{cases}
$$

PRoof. (3.17) follows from (3.16) and $\xi(T)=X$. Suppose (3.18) is not valid, then define

$$
T_{1}=\sup \{t>T ; \quad A<\xi(t)<B\} .
$$

Then at $T_{1}$, either $\xi\left(T_{1}\right)=A$ or $\xi\left(T_{1}\right)=B$. Without loss of generality, we can assume that $\xi\left(T_{1}\right)=B$. Then for $T<t<T_{1}, u_{A, B}$ is given by (3.19) and $A, B \in \operatorname{ch}\left(\xi\left(T_{1}\right), T_{1}\right)$. Therefore we have from (2.3)

$$
v\left(\xi\left(T_{1}\right), T_{1}\right)=\int_{A}^{B} u_{0, A, B}(\theta) d \theta+T_{1} f^{*}(0)=T_{1} f^{*}\left(\frac{\xi\left(T_{1}\right)-A}{T_{1}}\right)
$$

Hence from (3.15) and (3.21), we have $f^{*}(0)=f^{*}\left(\frac{\xi\left(T_{1}\right)-A}{T_{1}}\right)=f^{*}\left(\frac{B-A}{T_{1}}\right)$. Since $0=f^{*^{\prime}}\left(f^{\prime}(0)\right)=f^{*^{\prime}}(0)$ and therefore 0 is a critical point for $f^{*}$. This implies that $B-A=0$ which is a contradiction and this proves the Lemma.

Corollary 3.13. Let $f, u_{0, A, B}, u_{A, B}$ are as in Lemma 3.4. Furthermore assume that there exist $c>0$ and $0<\gamma<1$ such that for all $a<0<b$,

$$
f^{\prime^{-1}}(b)-f^{\prime^{-1}}(a) \geq c(b-a)^{\gamma}
$$

Then for all $t>0$

$$
\operatorname{TV}\left(u_{A, B}(., t)\right) \geq \min \left(\beta-\alpha, c\left(\frac{B-A}{t}\right)^{\gamma}\right)
$$

Proof. Let $0<t<T$, then from (3.16)

$$
u_{A, B}(\xi(t)-, t)-u_{A, B}(\xi(t)+, t)=\beta-\alpha .
$$


Let $t>T$, then from (3.19) and (3.22)

$$
\begin{aligned}
u_{A, B}(\xi(t)-, t)-u_{A, B}(\xi(t)+, t) & =f^{\prime^{-1}}\left(\frac{\xi(t)-A}{t}\right)-f^{\prime^{-1}}\left(\frac{\xi(t)-B}{t}\right) \\
& \geq c\left(\frac{B-A}{t}\right)^{\gamma} .
\end{aligned}
$$

This proves (3.23) and hence the corollary.

For proving (3) of the theorem (1.3), we need the following backward construction which was proved in [1] in obtaining the solution of control problems for conservation laws. Since the conservation law is irreversible, given a target function $g \in L^{\infty}$ at time $t=T>0$, in general there may not exist a $u_{0} \in L^{\infty}(\mathbb{R})$ such that the corresponding solution $u$ of (1.1) may not coincide with $g$ at $t=T$. This is mainly because, according to the Lax-Oleinik formula, $g$ must satisfy the compatibility condition, namely, $x \mapsto x-T f^{\prime}(g(x))$ is a nondecreasing function. Using the backward construction we can prove that it is also necessary. Basic idea of the proof is by discretization of the function $x-T f^{\prime}(g(x))$ and using the convexity assumption on $f$, we build the approximate solution and show that this solution converges and attains the value $g(x)$ at time $t=T$.

For the sake of completeness we will give the proof of this result in the appendix.

Let $\rho:\left[A_{1}, A_{2}\right] \rightarrow\left[A_{1}, A_{2}\right]$ be a non decreasing function. Let $t_{0}>0$ and

$$
\begin{aligned}
M & =\max _{x \in\left[A_{1}, A_{2}\right]} f^{\prime^{-1}}\left(\frac{x-\rho(x)}{t_{0}}\right) \\
\gamma_{1}(t) & =A_{1}+f^{\prime}(-M)\left(t-t_{0}\right) \\
\gamma_{2}(t) & =A_{2}+f^{\prime}(M)\left(t-t_{0}\right)
\end{aligned}
$$

Then we have the following.

LEMma 3.14 (Backward construction). Let $t_{0}>0, \rho,\left[A_{1}, A_{2}\right], \gamma_{1}$ and $\gamma_{2}$ are defined as above. Assume that $f^{\prime}(0)=0$, Then we have the following: There exist a $\bar{u}_{0} \in L^{\infty}\left(A_{1}, A_{2}\right)$ with $\left\|\bar{u}_{0}\right\|_{\infty}$ depends on $\rho, t_{0},\left(A_{2}-A_{1}\right)$. With this $u_{0}$, define

$$
u_{0, A_{1}, A_{2}}(x)=\left\{\begin{array}{lll}
0 & \text { if } & x \notin\left(A_{1}, A_{2}\right) \\
\bar{u}_{0}(x) & \text { if } & x \in\left(A_{1}, A_{2}\right) .
\end{array}\right.
$$


Let $u_{A_{1}, A_{2}}$ be the entropy solution of (1.1) with $u_{0, A_{1}, A_{2}}$ as its initial data. Then $u_{A_{1}, A_{2}}$ satisfies

(i) Let $0<t \leq t_{0}$, then

$$
u_{A_{1}, A_{2}}(x, t)=\left\{\begin{array}{lll}
0 & \text { if } & x \notin\left(A_{1}, A_{2}\right) \\
f^{\prime^{-1}}\left(\frac{x-\rho(x)}{t_{0}}\right) & \text { if } & x \in\left(A_{1}, A_{2}\right), t=t_{0}
\end{array}\right.
$$

(ii) Let $t>t_{0}$, then for $x \notin\left(\gamma_{1}(t), \gamma_{2}(t)\right), u_{A_{1}, A_{2}}(x, t)=0$.

Proof OF TheOREM 1.3. (1) Let $u_{0}$ be as in (3.1) and $u$ be the solution of (1.1). Let $F(t), \mu_{t}$ and $E_{t}$ be as in Lemma 3.11. From Lemma 3.9, $t \mapsto F(t)$ is a non decreasing function and hence there exist a countable set $S \subset(0, \infty)$ such that $F$ is continuous on $(0, \infty) \backslash S$. Therefore for all $t \notin S$, $F(t+)=F(t)$. Hence from (3.8) $\mu_{t}\left(E_{t}\right)=0$, for all $t \notin S$. Therefore from Lax-Olenik explicit formula $(2.9), x \mapsto f^{\prime}(u(x, t)) \in S B V(\mathbb{R})$ for all $t \notin S$. This proves (1).

(2) Let $\left\{b_{n}\right\} \in(0,1)$ be a sequence such that for $n \geq 2,0<b_{n}<b_{n-1}$ and $b_{n}<b_{n-1}-\frac{1}{n^{1 / \gamma}}$. Let $I_{n}=\left(b_{n-1}-\frac{1}{n^{1 / \gamma}}, \frac{1}{b_{n-1}}\right)$ and $u_{n}$ be the solution constructed in Lemma with $A_{n}=b_{n-1}-\frac{1}{n^{1 / \gamma}}, B_{n}=b_{n-1}, u_{0, n}=u_{0, A_{n}, B_{n}}$. Define

$$
\begin{aligned}
& u_{0}(x)= \begin{cases}u_{0, n}(x) & \text { if } x \in I_{n} \\
0 & \text { otherwise }\end{cases} \\
& u(x, t)= \begin{cases}u_{n}(x, t) & \text { if } x \in I_{n} \\
0 & \text { otherwise }\end{cases}
\end{aligned}
$$

In view of Rankine-Hugoniot condition, $u$ is the solution of (1.1) with data $u_{0}$. From corollary 3.2 , for any $t>0$,

$$
\begin{aligned}
T V(u(., t)) & \geq \sum_{n \geq 2} \min \left\{\beta-\alpha, c\left(\frac{B_{n}-A_{n}}{t}\right)^{\gamma}\right\} \\
& =\sum_{n \geq 2} \min \left\{\beta-\alpha, \frac{c}{t^{\gamma} n}\right\}=\infty .
\end{aligned}
$$

This proves (2).

(3) Let $0<h<\frac{1}{2}$ and $M \in \mathbb{R}$ be such that $\frac{(1-h)^{2}}{h}>f^{\prime}(M)>\frac{2 h}{1-2 h}$. Let $\gamma(\theta, t, A)=A+(\theta-t) f^{\prime}(M)$ and define $\left\{t_{n}\right\}$ and intervals $\left\{I_{n}=\left(A_{n}, B_{n}\right)\right\}$ 
as follows:

$$
\begin{gathered}
t_{0}=1, B_{0}=1 \quad A_{0}=1-h \\
t_{n+1}=t_{n}-h^{n+1}, B_{n+1}=\gamma\left(t_{n+1}, t_{n}, A_{n}\right), A_{n+1}=B_{n+1}-h^{n+1}
\end{gathered}
$$

Then as $n \rightarrow \infty$, we have

$$
\begin{aligned}
& t_{n+1}=1-h-h^{2}-h^{n+1} \rightarrow 1-\frac{h}{1-h}=\frac{1-2 h}{1-h}=t_{\infty} . \\
& A_{n+1}=A_{0}-\left(h+h^{2} \ldots+h^{n+1}\right) f^{\prime}(M)-h^{n+1} \rightarrow 1-h-\frac{h}{1-h} f^{\prime}(M)=A_{\infty} .
\end{aligned}
$$

Since $h<\frac{1}{2}$ and $\frac{(1-h)^{2}}{h}>f^{\prime}(M)$, we therefore have $t_{\infty}>0$ and $A_{\infty}>0$.

Let $\rho_{n}: I_{n} \rightarrow I_{n}$ be a non decreasing Cantor-Vitali function with $\rho_{n}\left(A_{n}\right)=A_{n}, \rho_{n}\left(B_{n}\right)=B_{n}$. Then from Lemma 3.5, there exist a $u_{0, n} \in$ $L^{\infty}(\mathbb{R})$ with support in $I_{n}$ such that the solution $u_{n}$ of (1.1) satisfies

$$
f^{\prime}\left(u_{n}(x, t)\right)= \begin{cases}0 & \text { if } \quad x \notin I_{n}, 0<t \leq t_{n} \\ \frac{x-\rho_{n}(x)}{t_{n}} & \text { if } \quad x \in I_{n}, t=t_{n} .\end{cases}
$$

Since $f^{\prime}(0)=0$, hence $u_{n}\left(x, t_{n}\right)=0$ for all $x \notin I_{n}$ and

$$
f^{\prime}\left(u_{n}\left(x, t_{n}\right)\right) \leq \max _{x \in I_{n}}\left|\frac{x-\rho_{n}(x)}{t_{n}}\right| \leq \frac{2 h_{n}}{t_{\infty}} \leq \frac{2}{t_{\infty}}<f^{\prime}(M) .
$$

Hence $u_{n}\left(x, t_{n}\right) \leq M$. Since $u_{n}\left(x, t_{n}\right)=0$ for $x>B_{n}$ and therefore from the method of characteristics, for $t>t_{n}, x>\gamma\left(t, t_{n}, B_{n}\right), u_{n}$ further satisfies

$$
u_{n}(x, t)=0
$$

Now define $u_{0} \in L^{\infty}(\mathbb{R})$ by

$$
u_{0}(x)= \begin{cases}u_{0, n}(x) & \text { if } x \in I_{n} \\ 0 & \text { otherwise }\end{cases}
$$

and let $u$ be the solution of (1.1). Then from (3.24) and (3.25), $u$ satisfies

$$
f^{\prime}(u(x, t))= \begin{cases}f^{\prime}\left(u_{n}(x, t)\right) & \text { if } \quad x \in I_{n}, t \in\left(0, t_{n}\right) \\ \frac{x-\rho_{n}(x)}{t} & \text { if } \quad x \in I_{n}, t=t_{n} .\end{cases}
$$

Since $\rho_{n} \notin S B V_{\text {loc }}(\mathbb{R})$, hence $f^{\prime}\left(u\left(x, t_{n}\right)\right) \notin S B V_{\text {loc }}(\mathbb{R})$ for all $n$. This proves (3) and hence the theorem. 


\section{Appendix}

Proof of Lemma 3.5. Let $C_{1}<C_{2}$ and $\rho_{0}$ be any non decreasing function. Then there exists $u_{0} \in L^{\infty}(\mathbb{R}), u \in L^{\infty}\left(\mathbb{R} \times \mathbb{R}_{+}\right)$such that $u$ is the solution of (1.1) with initial data $u_{0}$. Moreover u satisfies

$$
f^{p}(u(x, T))=\frac{x-\rho_{0}(x)}{T}
$$

Proof. Without loss of generality we can assume that $\rho_{0}:\left[C_{1}, C_{2}\right] \rightarrow$ $\left[C_{1}, C_{2}\right]$ is a non decreasing left continuous function with $\rho_{0}\left(C_{i}\right)=C_{i}$ for $i=1,2$.

Step 1: Discretization of $\rho_{0}$ : Let $n \geq 1$ and $C_{1}=y_{0}<y_{1}<$ $\cdots<y_{n}=C_{2}$ be such that $\left|y_{i}-y_{i+1}\right| \leq 1 / n$. From the left continuity, define $C_{1}=x_{0} \leq x_{1} \leq \cdots \leq x_{n}=C_{2}$ by $\rho_{0}^{-1}\left[y_{0}, y_{i}\right]=\left[x_{0}, x_{i}\right]$ and

$$
\rho_{n}(x)=\left\{\begin{array}{lll}
x & \text { if } & x \notin\left[C_{1}, C_{2}\right] \\
y_{0} \chi_{\left[y_{0}, y_{1}\right]}(x)+\sum_{i=1}^{n-1} y_{i} \chi_{\left(y_{i}, y_{i}+1\right]}(x) & \text { if } & x \in\left[C_{1}, C_{2}\right] .
\end{array}\right.
$$

Then for $x \in \mathbb{R}$,

$$
\left|\rho_{n}(x)-\rho_{0}(x)\right| \leq 1 / n .
$$

Step 2: Let $f\left(\theta_{f}\right)=\min _{x \in \mathbb{R}} f(x)$ and define $a_{i, n}, b_{i, n}, s_{i, n}, a_{i, n}(t), b_{i, n}(t), s_{i, n}(t)$, (See Figure 1) by

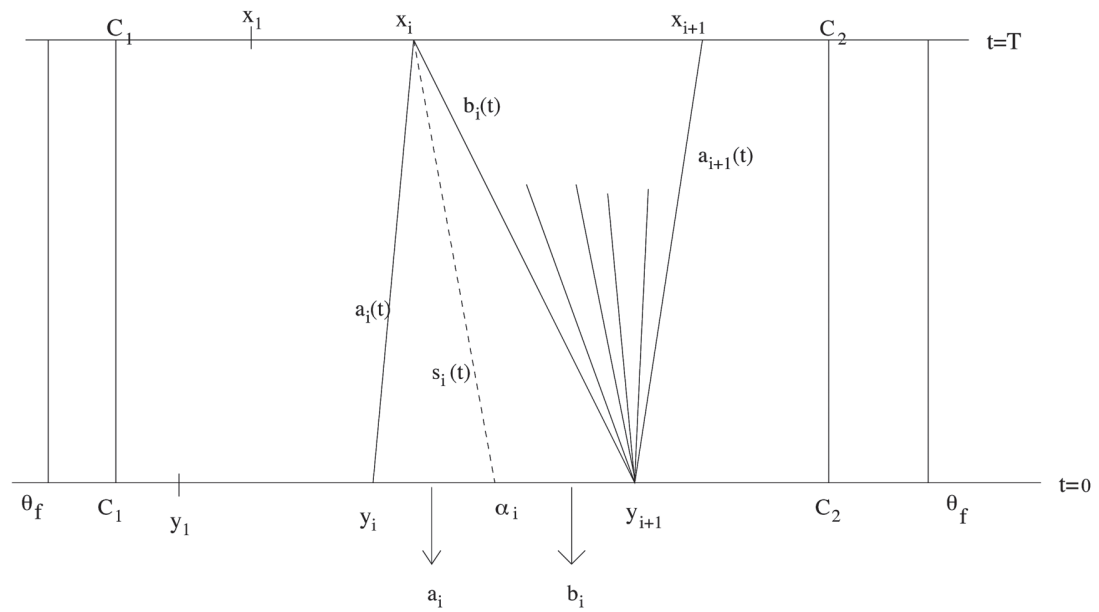

Figure 1. 


$$
\begin{aligned}
f^{\prime}\left(a_{i}, n\right) & =\frac{y_{i}-x_{i}}{T} \\
f^{\prime}\left(b_{i, n}\right) & =\frac{y_{i}-x_{i+1}}{T} \\
f^{\prime}\left(s_{i, n}\right) & =\frac{f\left(b_{i, n}\right)-f\left(a_{i, n}\right)}{b_{i, n}-a_{i, n}} \\
a_{i, n}(t) & =y_{i, n}+f^{\prime}\left(a_{i, n}\right)(t-T) \\
b_{i, n}(t) & =y_{i, n}+f^{\prime}\left(b_{i, n}\right)(t-T) \\
s_{i, n}(t) & =y_{i, n}+f^{\prime}\left(s_{i, n}\right)(t-T) .
\end{aligned}
$$

Now $f^{\prime}\left(a_{i, n}\right)=\frac{y_{i}-x_{i}}{T} \geq \frac{y_{i}-x_{i+1}}{T}=f^{\prime}\left(b_{i, n}\right) \quad$ and $\quad f^{\prime}\left(b_{i, n}\right)=\frac{y_{i}-x_{i+1}}{T} \leq$ $\frac{y_{i+1}-x_{i+1}}{T}=f^{\prime}\left(a_{i+1, n}\right)$. Hence $b_{i, n} \leq \min \left\{a_{i, n}, a_{i+1, n}\right\}$ and from convexity, $a_{i, n} \geq s_{i, n} \geq b_{i, n}$. Therefore for all $0 \leq t \leq T, a_{i, n}(t) \leq s_{i, n}(t) \leq b_{i, n}(t)$. Let $s_{i, n}(0)=\alpha_{i}$ and for $(x, t) \in \mathbb{R} \times[0, T]$, define

$$
f^{\prime}\left(u_{n}(x, t)\right)=\left\{\begin{array}{lll}
f^{\prime}\left(a_{i}, n\right) & \text { if } & a_{i, n}(t)<x<s_{i, n}(t) \\
f^{\prime}\left(b_{i}, n\right) & \text { if } & s_{i, n}(t)<x<b_{i, n}(t) \\
\frac{x_{i+1}-y}{t} & \text { if } & b_{i, n}(t)<x<a_{i+1, n}(t) .
\end{array}\right.
$$

Since $f^{\prime}\left(\theta_{f}\right)=0, a_{0, n}=a_{n, n}=\theta_{f}$, we have

$$
f^{\prime}\left(u_{n}(x, t)\right)=0 \quad \text { if } \quad x \notin\left[C_{1}, C_{2}\right], 0 \leq t \leq T .
$$

Then clearly $u_{n}$ satisfies

$$
\begin{aligned}
& u_{n_{t}}+f\left(u_{n}\right)_{x}=0 \quad \mathbb{R} \times(0, T) \\
& f^{\prime}\left(u_{n}(x, T)\right)= \begin{cases}\frac{x-\rho_{n}(x)}{T} & \text { if } C_{1}<x<C_{2} \\
0 & \text { if } x \notin\left[C_{1}, C_{2}\right] .\end{cases}
\end{aligned}
$$

Also

$$
u_{n}(x, 0)=\left\{\begin{array}{lll}
a_{i, n} & \text { if } & a_{i, n}<x<s_{i, n}(0) \\
b_{i, n} & \text { if } & s_{i, n}(0)<x<a_{i+1, n} \\
\theta_{f} & \text { if } & x \notin\left[C_{1}, C_{2}\right]
\end{array}\right.
$$

Hence

$$
\begin{aligned}
\left|f^{\prime}\left(u_{n}(x, 0)\right)\right| & \leq \operatorname{Max}\left\{\left|f^{\prime}\left(a_{i}, n\right)\right|,\left|f^{\prime}\left(b_{i}, n\right)\right|\right\} \\
& \leq \frac{\left|C_{2}-C_{1}\right|}{T}
\end{aligned}
$$


and

$$
\begin{aligned}
T V\left(f^{\prime}\left(u_{n}(x, 0)\right)\right) & \leq \sum\left|f^{\prime}\left(a_{i}, n\right)-f^{\prime}\left(b_{i}, n\right)\right|+\sum\left|f^{\prime}\left(b_{i}, n\right)-f^{\prime}\left(a_{i+1, n}\right)\right| \\
& =\sum\left|\frac{x_{i}-x_{i+1}}{T}\right|+\sum\left|\frac{y_{i}-y_{i+1}}{T}\right| \\
& \leq \frac{2\left(C_{2}-C_{1}\right)}{T} .
\end{aligned}
$$

Hence from Helley's theorem, there exist a subsequence still denoted $\left\{f^{\prime}\left(u_{n}(\cdot, 0)\right)\right\}$ which converges to a function $z$ pointwise. Let $u_{0}(x)=$ $\left(f^{\prime}\right)^{-1}(z(x))$, hence $u_{n}(x, 0) \rightarrow u_{0}(x)$. From super linear growth, $\left\{u_{n}(\cdot, 0)\right\}$ are uniformly bounded, hence $u_{0} \in L^{\infty}(\mathbb{R})$. Further more by construction, $u_{n}$ satisfies

$$
f^{\prime}\left(u_{n}(x, T)\right)=\frac{x-\rho_{n}(x)}{T} .
$$

Let $u$ be the solution of (1.1) with $u_{0}$ as its initial data. From the dominated convergence Theorem, $u_{n}(\cdot, 0) \rightarrow u_{0}$ in $L_{l o c}^{1}(\mathbb{R})$, hence from $L^{1}$ contractivity, $u_{n}(x, T) \rightarrow u(x, T)$ a.e. $x \in \mathbb{R}$. Therefore for a.e. $x \in \mathbb{R}$,

$$
f^{\prime}(u(x, T))=\frac{x-\rho_{0}(x)}{T} .
$$

This proves the Lemma.

In order to prove Lemma 3.10, we need the following covering lemma (see [11]).

LEMMA 4.1. Let $I=(a, b)$ and $\mu$ be a non negative finite measure on I. Let $E \subset I$ and $\varepsilon_{0}>0$ such that $\mu^{*}(E) \geq \varepsilon_{0}$, where $\mu^{*}$ denotes the outer measure. Let $\Re$ be a family of open intervals covering $E$. Then there exists a sub family $\Re_{1} \subset \Re$ which consists of mutually disjoint open intervals such that

$$
\sum_{J \in \Re_{1}} \mu(J) \geq \frac{\varepsilon_{0}}{4}
$$

Proof. Let $E \subset \cup_{J \in \Re} J=\cup_{n=1}^{\infty}\left(a_{n}, b_{n}\right)$, with $\left(a_{n}, b_{n}\right) \cap\left(a_{m}, b_{m}\right)=\phi$ for $n \neq m$. Let $\varepsilon_{n}=\mu\left(\left(a_{n}, b_{n}\right)\right)$, then

$$
\varepsilon_{0} \leq \mu^{*}(E) \leq \sum_{n=1}^{\infty} \mu\left(\left(a_{n}, b_{n}\right)\right)=\sum_{n=1}^{\infty} \varepsilon_{n} .
$$


Choose $\left[\alpha_{n}, \beta_{n}\right] \subset\left(a_{n}, b_{n}\right)$ such that $\mu\left(\left[\alpha_{n}, \beta_{n}\right]\right) \geq 3 \frac{\varepsilon_{n}}{4}$. Since $\Re$ is a cover of $\left[\alpha_{n}, \beta_{n}\right]$ and hence there exists a finite number of open sets $\left\{J_{i}^{(n)}\right\} \subset \Re$ such that $\left[\alpha_{n}, \beta_{n}\right] \subset \cup J_{i}^{(n)} \subset\left(a_{n}, b_{n}\right)$. By discarding some of the sets if necessary, we can assume that for each $i, J_{i}^{(n)}$ is not contained in $\cup_{k \neq i} J_{k}^{(n)}$. Let $x_{i} \in J_{i}^{(n)} \backslash \cup_{k \neq i} J_{k}^{(n)}$ and by reordering of the indicies we can assume that $a_{n}<x_{1}<x_{2}<\cdots<b_{n}$. Hence $G_{e}^{(n)}=\left\{J_{i}^{(n)}: i\right.$ is even $\}$ and $G_{o}^{(n)}=$ $\left\{J_{i}^{(n)}: i\right.$ is odd $\}$ consists of disjoint sets and

$$
\sum_{i \in G_{e}^{(n)}} \mu\left(J_{i}^{(n)}\right)+\sum_{i \in G_{o}^{(n)}} \mu\left(J_{i}^{(n)}\right) \geq \mu\left(\left[\alpha_{n}, \beta_{n}\right]\right) \geq 3 \frac{\varepsilon_{n}}{4} .
$$

Choose $G^{(n)}$ to be one of the family $G_{e}^{(n)}$ or $G_{o}^{(n)}$ such that

$$
\sum_{i \in G^{(n)}} \mu\left(J_{i}^{(n)}\right) \geq 3 \frac{\varepsilon_{n}}{8} \geq \frac{\varepsilon_{n}}{4} .
$$

Let $\Re_{1}=\cup_{n=1}^{\infty} G^{(n)}$, then $\Re_{1} \subset \Re$ consists of disjoint open intervals and

$$
\sum_{J \in \Re_{1}} \mu(J) \geq \sum_{n} \mu\left(G^{(n)}\right) \geq \frac{1}{4} \sum \varepsilon_{n} \geq \frac{\varepsilon_{0}}{4} .
$$

This proves the Lemma.

LEMMA 4.2. Let $\mu, \gamma$ be non negative mutually singular measures on $(a, b)$. Let $D(\mu)$ and $D(\gamma)$ be the points of discontinuities of $\mu([a, x])$ and $\gamma([a, x])$ respectively. Let $E \subset(a, b) \backslash(D(\mu) \cup D(\gamma))$ be a measurable set with $\mu(E)>0$ and $\gamma(E)=0$. Then for $\mu$ a.e. $x \in E$

$$
\lim _{h \rightarrow 0} \frac{\mu((x, x+h])}{\gamma((x, x+h])}=\lim _{h \rightarrow 0} \frac{\mu((x-h, x])}{\gamma((x-h, x])}=\infty .
$$

Proof. Let $N>0$ and define

$$
E_{N}=\left\{x \in E: \varlimsup_{h \rightarrow 0} \frac{\mu((x, x+h])}{\gamma((x, x+h])}<N\right\} .
$$

Claim: $\mu\left(E_{N}\right)=0$. Suppose not and let $\mu\left(E_{N}\right) \geq \varepsilon_{0}>0$ for some $\varepsilon_{0}$. Let $E_{N} \subset U$ be an open set. Then for $x \in E_{N}$, choose $h_{n}>0$ such that $\left[x, x+h_{n}\right] \subset U$ and $\mu\left(\left(x, x+h_{n}\right]\right)<N \gamma\left(\left(x, x+h_{n}\right]\right)$. Since $x \notin D(\mu) \cup D(\gamma)$ and hence by continuity, choose $a(x)<x<b(x)$ such that $I(x)=$ $(a(x), b(x)) \subset U$ and $\mu((a(x), b(x))) \leq N \gamma((a(x), b(x)))$. Let $\Re=\{I(x)\}_{x \in E_{N}}$ is a cover of $E_{N}$ and hence by lemma 4.1 there exists a subfamily $\Re_{1}$ of 
$\Re$ such that

$$
\begin{aligned}
\frac{\varepsilon_{0}}{4} \leq \sum_{J \in \Re_{1}} \mu(J) & \leq N \sum_{J \in \Re_{1}} \gamma(J) \\
& =N \gamma\left(\cup_{J \in \Re_{1}} J\right) \\
& \leq N \gamma(U) .
\end{aligned}
$$

Hence $\frac{\varepsilon_{0}}{4} \leq N \inf \left\{\gamma(U): E_{N} \subset U\right\}=0$, since $\gamma(E)=0$. This is a contradiction and hence the claim. Since $\left\{x \in E: \underline{\lim _{h \rightarrow 0}} \frac{\mu((x, x+h])}{\gamma((x, x+h])}<\infty\right\}=\cup_{N=1}^{\infty} E_{N}$ and
hence for $\mu$ a.e. $x \in E$

$$
\lim _{h \rightarrow 0} \frac{\mu((x, x+h])}{\gamma((x, x+h])}=\infty .
$$

This proves the Lemma.

\section{Proof of Lemma 3.2:}

Proof. Take $\mu=\mu_{t}, \gamma=d x, E=E_{t}$ in Lemma 4.2. Since for $x \in E_{t}$, $x-h \notin D(\mu), \mu((x, x-h]) \leq y_{+}(x, t)-y_{+}(x-h, t)$ and hence the Lemma.

\section{REFERENCES}

[1] Adimurthi - S.S. Ghoshal - G.D. Veerappa Gowda, Structure of the entropy solution of a scalar conservation law with strict convex flux, J. Hyperbolic Differ. Equ. 9 (2012), no. 4, 571-611.

[2] Adimurthi - S.S. Ghoshal - G.D. Veerappa Gowda, Exact controllability of scalar conservation law with strict convex flux, Math. Control Relat. Fields 4 (2014), no. 4.

[3] Adimurthi - S.S. Ghoshal - G.D. Veerappa Gowda, Optimal controllability for scalar conservation law with convex flux, J. Hyperbolic Differ. Equ. 11 (2014), no. 3, 477-491.

[4] L. Ambrosio - N. Fusco - D. Pallara, Functions of bounded variation and free discontinuity problems. Oxford Mathematical Monographs. The Clarendon Press, Oxford University Press, New York, 2000.

[5] L. Ambrosio - C. DE Lellis, A note on admissible solutions of $1 D$ scalar conservation laws and $2 D$ Hamilton-Jacobi equations. J. Hyperbolic Differ. Equ. 1 (2004), no. 4, 813-826.

[6] S. BIANCHINI - D. Tonon, SBV-like regularity for Hamilton-Jacobi equations with a convex Hamiltonian, J. Math. Anal. Appl. 391 (2012), no. 1, 190-208.

[7] S. Bianchini, SBV regularity for scalar conservation laws, Preprint. 
[8] S. Bianchini - C. De Lellis - R. Robyr, SBV regularity for Hamilton-Jacobi equations in Rn. Arch. Ration. Mech. Anal. 200 (2011), no. 3, 1003-1021.

[9] S. BIANCHINI, SBV regularity of genuinely nonlinear hyperbolic systems of conservation laws in one space dimension. Acta Math. Sci. Ser. B Engl. Ed. 32 (2012), no. 1, 380-388.

[10] S. Bianchini - L. CARAVEnna, SBV regularity for genuinely nonlinear, strictly hyperbolic systems of conservation laws in one space dimension. Comm. Math. Phys. 313 (2012), no. 1, 1-33.

[11] M.W. Botsko, An elementary proof of Lebesgue differentiation theorem, Amer. Math. Monthly 110 (2003), no. 9, 834-838.

[12] L.C. Evans, Partial differential equations, Graduate studies in Mathematics, vol. 19, AMS 1998.

[13] L.C. Evans - R.F. GARIEPY, Measure theory and fine properties of functions, CRC Press, 1992.

[14] S. N. KRUZKov, First order quasilinear equations with several independent variables. Mat. Sb. (N.S.) 81 (123), 1970, 228-255; English translation in: Math. USSR-Sb. 10 (1970), no. 2, 217-243.

[15] R. RoBYR, SBV regularity of entropy solutions for a class of genuinely nonlinear scalar balance laws with non-convex flux function. J. Hyperbolic Differ. Equ. 5 (2008), no. 2, 449-475.

[16] D. Tonon, Some applications of the SBV Regularity Theorem for entropy solutions of $1 D$ scalar conservation laws to convection theory and sticky particles, Riv. Mat. Univ. Parma (N.S.) 3 (2012), no. 1, 163-175.

Manoscritto pervenuto in redazione il 13 Novembre 2012. 